\section{P2-331 NON-STEROIDAL ANTI-INFLAMMATORY DRUG AND ASPIRIN USE AND THE RISK OF HEAD AND NECK CANCER: A SYSTEMATIC REVIEW}

doi:10.1136/jech.2011.142976k.63

\section{P2-333 ACUTE MYOCARDIAL INFARCTION: INCIDENCE AND 30-DAY CASE FATALITY IN ENGLAND FOR FIRST AND RECURRENT EVENTS}

doi:10.1136/jech.2011.142976k.65

J C Wilson, ${ }^{*}$ L Anderson, L Murray, C Hughes. Queen's University Belfast, Belfast, UK

Introduction Use of non-steroidal anti-inflammatory drugs (NSAIDs) has been associated with a reduced risk of several cancers. We conducted a systematic literature review to investigate the association between NSAID/aspirin use and risk of head and neck cancer (HNC).

Method Medline, Embase, PubMed, Cochrane Library and Web of Science were systematically searched using terms for NSAIDs/ aspirin, HNC and observational/intervention study designs.

Results Of 9268 articles identified, two population-based prescribing database studies and three case-control studies met the selection criteria. The studies investigated different HNC sites. Only one study found a significant protective effect of aspirin use for HNC risk (OR $0.75,95 \%$ CI 0.58 to 0.96 ) and one showed a significantly increased risk of oral/oropharangeal cancer with non-low-dose aspirin NSAID use (OR 3.5, 95\% CI 1.8 to 6.7).

Conclusion No definitive conclusion on the effect of NSAIDs/aspirin on HNC risk was possible. Further robust large-scale studies are required to clarify any possible association.

\section{P2-332 SELECTING PRIORITY HEALTH RISK FACTORS FOR RESEARCHING PREVENTIVE INTERVENTIONS: A NEW ZEALAND EXAMPLE}

doi:10.1136/jech.2011.142976k.64

${ }^{1} \mathrm{~N}$ Wilson, ${ }^{*}{ }^{1} \mathrm{~T}$ Blakely, ${ }^{1} \mathrm{R}$ Foster, ${ }^{1} \mathrm{D}$ Hadorn, ${ }^{2} \mathrm{~T}$ Vos. ${ }^{1}$ University of Otago, Wellington, Wellington, New Zealand; ${ }^{2}$ University of Queensland, Brisbane, Australia

Introduction To maximise health gain with constrained health sector resources, it is necessary to identify the highest priority risk factor areas for studying preventive interventions. We aimed to develop a process for prioritising such risk factors for further research, in the New Zealand setting.

Methods Using WHO data for high-income countries in the Western Pacific Region, the burden of disease in disability-adjusted life years associated with leading risk factors was used as a starting point. Subsequent prioritising steps included: the existence of effective and (likely) cost-effective preventive interventions for each risk factor, and the contribution of the risk factor to health inequalities.

Results The top six major risk factors identified were: tobacco use, high blood pressure, high cholesterol, alcohol use, overweight/ obesity and physical inactivity. All of these six risk factors contribute to ethnic health inequalities (Maori vs non-Maori). They are also all relevant to reducing the health burden for children/youth and older adults, and four were relevant to reducing health inequalities for socio-economically deprived New Zealanders. For all of the top six risk factor areas there are published studies indicating that one or more preventive interventions are cost-saving (to the health sector or society).

Conclusions This process identified risk factor areas associated with high health burden and which are amenable to cost-effective preventive interventions. Our next step is to work with stakeholders to select the range of interventions within each risk factor area that are of most interest for cost-effectiveness analysis.
Introduction There are several population-based English studies of AMI, but they are local rather than national, do not necessarily distinguish first events and re-infarctions, and are out of date. Our objective was to study AMI incidence and 30-day case fatality rates (CFRs) for first and recurrent events in England in 2007 (population 51.8 million).

Methods Analysis of linked routine national hospital and mortality data, with an 8-year washout period to identify people who already had a prior event, in order to distinguish whether each AMI in 2007 was first or recurrent.

Results Of 88311 AMI events in 2007, 86\% were first events. Agestandardised incidence of first AMI (per 100000 person years) was 151 (150-152) in men and 64 (63-65) in women. Age-standardised 30-day overall CFRs including sudden deaths without hospitalisation for first AMI were 35.5\% (35.1-36.0) and 34.4\% (33.3-35.4) in men and $34.4 \%(33.9-35.0)$ and $31.0 \%$ (29.8-32.3) in women, for first AMIs and re-infarctions, respectively. Age-standardised 30-day hospitalised CFRs were much lower at $14.5 \%(14.1-14.9)$ and $14.1 \%$ $(13.2-15.0)$ in men and $15.2 \%(14.7-15.7)$ and $13.9 \%(12.8-15.0)$ in women for first AMIs and re-infarctions, respectively.

Conclusions This study provides, for the first time, total nationallevel population-based estimates of first and recurrent AMI as well as overall AMI incidence and case fatality in England. It holds informative value in better understanding AMI epidemiology and prognosis that should prove useful to practitioners and policy makers.

\section{P2-334 ALCOHOL AND MORTALITY AMONG 220000 MEN IN CHINA 1990-2005: A NATIONALLY REPRESENTATIVE PROSPECTIVE STUDY}

doi:10.1136/jech.2011.142976k.66

${ }^{1} \mathrm{~L}$ Yang, ${ }^{*}{ }^{2} \mathrm{M}$ Zhou, ${ }^{1} \mathrm{P}$ Sherliker, ${ }^{2} \mathrm{G}$ Yang, ${ }^{1} \mathrm{R}$ Peto, ${ }^{2} \mathrm{~J}$ Wang, ${ }^{1} \mathrm{I}$ Millwood, ${ }^{2} \mathrm{Y} \mathrm{Hu}$, ${ }^{1} \mathrm{M}$ Smith, ${ }^{1} \mathrm{Z}$ Chen. ${ }^{1}$ University of Oxford, Oxford, UK; ${ }^{2}$ Chinese Center for Disease Control and Prevention, Beijing, China

Introduction Light-to-moderate alcohol consumption is associated with decreased mortality from cardiovascular diseases in most studies in the West. Data on the health effects of alcohol drinking in China, which involves mainly distilled spirit, are limited.

Methods A nationally representative prospective cohort study included 220000 Chinese men aged 40-79 years who were recruited in 1990-1991 from 45 widely separated study sites. By 1 January 2006, over 40000 deaths had been recorded at age 40-80 years. Cox regression was used to relate alcohol drinking to cause-specific mortality, adjusting simultaneously for age, area, smoking and education.

Results About a third of participants reported drinking alcohol regularly (ie, drank at least once a week), mainly involving spirit. The estimated mean alcohol consumption among regular drinkers was $372 \mathrm{~g} /$ week. There was a J-shaped association between amount of alcohol consumed and all-cause mortality, after excluding all men with prior disease at baseline and the first 3 years of follow-up, with those who drank $<140 \mathrm{~g} /$ week having the lowest overall mortality. Regular alcohol drinking was hardly related to respiratory mortality, but J-shaped associated with mortality from IHD, stomach cancer and lung cancer, and positively associated with mortality from stroke, oesophageal cancer and liver cirrhosis and
K Smolina, ${ }^{*}$ M Goldacre, L Wright, M Rayner. University of Oxford, Oxford, UK 
accidental deaths (eg, violence, suicide and trauma). There is no apparent evidence of reduced mortality from CVD among lightto-moderate drinkers.

Conclusion Among Chinese adults aged 40-79 there was a J-shaped relationship between alcohol consumption and overall mortality.

\section{P2-335 CANCER MORTALITY AMONG CHRYSOTILE WORKERS}

doi:10.1136/jech.2011.142976k.67

${ }^{1} \mathrm{E}$ Yano, ${ }^{2} \mathrm{X}$ Wang, ${ }^{3} \mathrm{Z}-\mathrm{M}$ Wang, ${ }^{3} \mathrm{M}-\mathrm{Z}$ Wang, ${ }^{3} \mathrm{Y}$-J Lan. ${ }^{1}$ Teikyo University School of Public Health, Tokyo, Japan, ${ }^{2}$ School of Public Health and Primary Care, The Chinese University of Hong Kong, Hong Kong, China; ${ }^{3}$ West China Center of Medical Sciences, Sichuan University, Chengdu, China

Since the beginning of 1972, we have followed a group of workers in an asbestos plant in Chongqing, China. In the plant, chrysotile has been used to manufacture asbestos textile, asbestos cement, flooring materials, friction materials, etc. Previously, we performed a matched (1:5) case-control study by selecting cases of workers who developed lung cancer sometime between 1975 and 2001 and found the elevated risk of lung cancer associated with the exposure. In 2006, we summarised further follow-up results of the cohort and found that, in addition to the 132 deaths until the end of 25 th year, 101 workers were deceased during the last 10 years, and among them, 29 were from lung cancer (total: 51 lung cancers during the 35 years). Depending on the concentration of fibre of their workplace, we divided the workers into three levels of exposure. Compared to those in the administration and asbestos cement sections, workers in raw material and textile departments showed a higher HR for lung cancer $(3.9,95 \%$ CI 1.8 to 8.5$)$ in Cox proportional hazard model analysis. Similarly, HRs for total cancer and total death were 2.0 (1.2 to 3.3) and 1.5 (1.1 to 2.0), respectively. Multivariate analysis controlling for age, smoking, and working time did not change the results greatly. The results of the present study confirm the strong association of chrysotile exposure and lung cancer. Results of the fibre type analysis of asbestos would be presented.

\section{P2-336 FACTORS ASSOCIATED WITH CLASS III OBESITY AMONG ADULTS, NATIONAL TELEPHONE SURVEY (VIGITEL) BRAZIL, 2009}

doi:10.1136/jech.2011.142976k.68

\begin{abstract}
${ }^{1,2} \mathrm{R}$ Yokota, ${ }^{*}{ }^{1} \mathrm{~B}$ Iser, ${ }^{1} \mathrm{~L}$ Sardinha, ${ }^{1} \mathrm{~L}$ Moura, ${ }^{3} \mathrm{R}$ Claro, ${ }^{1} \mathrm{D}$ Malta. ${ }^{1}$ General Coordination of Non Communicable Diseases and Injuries, Ministry of Health, Brasilia, Distrito Federal, Brazil; ${ }^{2}$ Brazilian Field Epidemiology Training Program, Ministry of Health, Brasilia, Distrito Federal, Brazil; ${ }^{3}$ Center for Epidemiological Studies in Health and Nutrition, University of São Paulo, Sao Paulo, Sao Paulo, Brazil
\end{abstract}

Introduction In Brazil, class III obesity has increased from $0.18 \%$ (1975), to $0.33 \%$ (1989) and $0.64 \%$ (2003) in the last decades, according to National surveys. However, no information on recent trends in class III obesity prevalence is available in Brazil.

Objective To estimate the prevalence of class III obesity and its associations with sociodemographic and behavioural factors. Method: We analysed data from the Telephone-based Surveillance System of Risk and Protective Factors for Chronic Diseases (Vigitel) collected in 2009. Approximately 54000 adults were interviewed in all al state capitals and federal district. Individuals with body mass index higher than $40 \mathrm{~kg} / \mathrm{m}^{2}$ were identified as class III obese. Poisson regression models were used to evaluate the factors associated with class III obesity.

Result The prevalence of class III obesity was $1.1 \%$ ( $n=489 ; 95 \%$ CI 0.9 to $1.3 \%$ ) in 2009. Class III obesity was associated with sex $(\mathrm{PR}=2.36$; $95 \% \mathrm{CI} 1.4$ to 3.8$)$, dyslipidemia ( $\mathrm{PR}=1.9 ; 95 \% \mathrm{CI} 1.3$ to
2.8), hypertension ( $\mathrm{PR}=5.5 ; 95 \%$ CI 3.6 to 8.3 ), diabetes $(\mathrm{PR}=1.8$; $95 \%$ CI 1.2 to 2.7$)$, cardiovascular disease ( $\mathrm{PR}=2.2 ; 95 \% \mathrm{CI} 1.0$ to 4.6), watching television more than 3 hours/day ( $\mathrm{PR}=1.8 ; 95 \% \mathrm{CI}$ 1.1 to 3.0 ) and being current smoker ( $\mathrm{PR}=0.5$; $95 \%$ CI 0.3 to 0.8 ). When we included all associated factors in the model, sex $(\mathrm{PR}=2.0$; $95 \% \mathrm{CI}-1.2$ to -3.3 ; $\mathrm{p}$-value $=0.004)$, hypertension $(\mathrm{PR}=5.3 ; 95 \%$ CI 3.0 to 9.5 ; p-value $<0.001)$, watching television more than 3 hours/day ( $\mathrm{PR}=1.8 ; 95 \% \mathrm{CI} 1.1$ to 2.9 ; $\mathrm{p}$-value $=0.020$ ) and being current smoker $(\mathrm{PR}=0.5 ; 95 \%$ CI 0.2 to $0.8 ; \quad p$-value $=0.012)$ remained associated with class III obesity.

Conclusion Vigitel is an efficient tool to monitor class III obesity in Brazil. This study identified some factors associated with class III obesity that should be considered when developing prevention measures due to its relations with several chronic diseases.

\section{P2-337 SHORT TERM TRENDS OF OBESITY USING DATA OF THE TELEPHONE-BASED SURVEILLANCE SYSTEM OF RISK AND PROTECTIVE FACTORS FOR CHRONIC DISEASES (VIGITEL), BRAZIL, 2006 TO 2009}

doi:10.1136/jech.2011.142976k.69

${ }^{1,2} \mathrm{R}$ Yokota, ${ }^{*} \mathrm{~B}$ Iser, ${ }^{1} \mathrm{~L}$ Sardinha, ${ }^{1} \mathrm{~L}$ Moura, ${ }^{1,3} \mathrm{R}$ Claro, ${ }^{1} \mathrm{D}$ Malta. ${ }^{1}$ General Coordination of Non Communicable Diseases and Injuries, Ministry of Health, Brasilia, Distrito Federal, Brazil; ${ }^{2}$ Brazilian Field Epidemiology Training Program, Brasilia, Distrito Federal, Brazil; ${ }^{3}$ Center for Epidemiological Studies in Health and Nutrition, University of São Paulo, São Paulo, São Paulo, Brazil

Introduction Obesity has increased in high and low income countries, among all age groups. In Brazil, obesity has tripled in men and doubled in women from 1975 to 2003. Objective: To analyse short term trends in the prevalence of obesity among Brazilian adults from 2006 to 2009. Method: Data from Vigitel collected annually from 2006 to 2009 were analysed. Poisson regression models with obesity as the dependent variable and the year of the study as the explanatory variable were used to identify linear trends.

Result Obesity prevalence in Brazil increased from 11.4\% (95\% CI 10.8 to $12.0 \%$ ) in 2006 to $13.9 \%$ (95\% CI 13.1 to $14.7 \%$, p-value $<0.001)$ in 2009, without a significant difference between sex (2006 - female: $11.4 \%$, 95\% CI 10.6 to $12.2 \%$; male: $11.4 \%$; $95 \%$ CI 10.5 to $12.3 \%$; 2009 - female: $14.0 \%, 95 \%$ CI 13.1 to $14.0 \%$; male: $13.7 \%$; $95 \%$ CI 12.4 to $15.0 \%$ ). The higher prevalence of obesity was observed among adults with less educational level in all years studied (0-8 years of schooling: $2006-13.6 \%, 95 \%$ CI 12.5 to $14.6 \%$; $2009-16.1 \%$, $95 \%$ CI 14.6 to $17.6 \%$, p-value $=0.004 ; 9-11$ years of schooling: $2006-8.9 \%, 95 \%$ CI 8.2 to $9.6 \% ; 2009-11.6 \%, 95 \%$ CI 10.7 to $12.4 \%$, p value $<0.001$; $\geq 12$ years of schooling: $2006-9.6 \%$, $95 \%$ CI 8.6 to $10.6 \%$; 2009: 11.6 , $95 \%$ CI 10.6 to $12.6 \%$, $\mathrm{p}$ value $=0.007$ )

Conclusion The obesity increase among Brazilian adults demonstrates the need to improve prevention measures in Brazil. The agile and inexpensive data collection method used allowed it to have an important role in obesity plan control recently developed in Brazil.

\section{P2-338 ASSOCIATION OF BODY MASS INDEX WITH RISK OF AGE-RELATED CATARACTS IN A MIDDLE-AGED JAPANESE POPULATION}

doi:10.1136/jech.2011.142976k.70

${ }^{1} \mathrm{M}$ Yoshida, ${ }^{*} \mathrm{~K}$ Karita, ${ }^{2} \mathrm{M}$ Inoue, ${ }^{2} \mathrm{M}$ Imasaki, ${ }^{2} \mathrm{~S}$ Tsugane. ${ }^{1}$ Department of Public Health, Kyorin University School of Medicine, Mitaka-shi, Tokyo, Japan; ${ }^{2}$ Epidemiology and Preventive Division, Research Center for Cancer Prevention and Screening, National Cancer Center, Chuo-ku, Tokyo, Japan

Introduction Many epidemiological studies have demonstrated that body mass index (BMI) is associated with the risk of developing 